\title{
Propensity score-matching analysis to compare clinical outcomes of endoscopic submucosal dissection for early gastric cancer in the postoperative and non- operative stomachs
}

\author{
Mitsuru Esaki ${ }^{1,2^{*}}$ (D, Sho Suzuki ${ }^{1}$, Yasuyo Hayashi ${ }^{2,3}$, Azusa Yokoyama ${ }^{2}$, Shuichi Abe ${ }^{2}$, Taizo Hosokawa², \\ Shinichi Tsuruta ${ }^{4}$, Yosuke Minoda ${ }^{3}$, Yoshitaka Hata ${ }^{3}$, Haruei Ogino ${ }^{3}$, Hirotada Akiho ${ }^{2}$, Eikichi Ihara ${ }^{3}$ \\ and Yoshihiro Ogawa ${ }^{3}$
}

\begin{abstract}
Background: Endoscopic submucosal dissection (ESD) of the postoperative stomach (ESD-P) for early gastric cancer (EGC) is considered a technically difficult procedure. However, it is difficult to compare the outcomes of ESD-P and ESD of the non-operative stomach (ESD-N) because their baseline characteristics are different. Therefore, we aimed to compare the technical outcomes of ESD-P with those of ESD-N using a propensity score-matching analysis to compensate for the differences.

Methods: The chart records of 1046 patients with EGC who were treated with ESD between January 2004 and July 2016 at Kitakyushu Municipal Medical Center in Japan were reviewed in this retrospective study. Multivariate analyses and propensity score-matching were performed for age, sex, lesion location, lesion size, tumor invasion, tumor size, ulcer (scar), and operator skill. The primary outcome was procedure time. Secondary outcomes were percentages of en bloc, complete, and curative resections, and percentages of adverse events, which were evaluated between the two groups.

Results: Forty-one patients were in the ESD-P group and 1005 patients were in the ESD-N group. Propensity score-matching created 41 matched pairs. According to the adjusted comparisons, ESD-P required a significantly longer procedure time (85 min vs 51 min, $p<0.001$ ). Other treatment outcomes showed an en bloc resection rate of 100\% for both groups $(p=1)$ and complete resection rates of 95.1 and $97.6 \%(p=1)$, curative resection rates of 90.2 and $90.2 \%(p=1)$, perforation during ESD rates of 2.4 and $0 \%(p=1)$, and postprocedure bleeding rates of 2.4 and $2.4 \%(p=1)$ for the ESD-P and ESD-N groups, respectively. For the ESD-P group, lesions on the suture line or anastomotic site were significantly associated with longer procedure times $(p=0.038)$.

(Continued on next page)
\end{abstract}

\footnotetext{
* Correspondence: esaki_saiseikai@yahoo.co.jp

${ }^{1}$ Division of Gastroenterology and Hepatology, Department of Medicine,

Nihon University School of Medicine, 1-6 Kanda-Surugadai, Chiyoda-ku,

Tokyo 101-8309, Japan

${ }^{2}$ Department of Gastroenterology, Kitakyushu Municipal Medical Center,

2-1-1 Bashaku, Kokurakita-ku, Kitakyushu, Fukuoka 802-0077, Japan

Full list of author information is available at the end of the article
}

(c) The Author(s). 2018 Open Access This article is distributed under the terms of the Creative Commons Attribution 4.0 International License (http://creativecommons.org/licenses/by/4.0/), which permits unrestricted use, distribution, and

reproduction in any medium, provided you give appropriate credit to the original author(s) and the source, provide a link to the Creative Commons license, and indicate if changes were made. The Creative Commons Public Domain Dedication waiver (http://creativecommons.org/publicdomain/zero/1.0/) applies to the data made available in this article, unless otherwise stated. 
(Continued from previous page)

Conclusions: ESD-P was a more time-consuming procedure than ESD-N. However, ESD-P and ESD-N achieved high rates of curative resection with a low rate of adverse events for the treatment of EGC. ESD could be selected as the treatment for EGC even in the postoperative stomach provided that careful attention is given to lesions on the suture line or anastomotic site.

Keywords: Endoscopic submucosal dissection, Postoperative stomach, Gastric cancer

\section{Background}

Total and subtotal gastrectomies have been performed for the treatment of gastric cancer and benign peptic ulcers [1]. In addition, esophagectomy with reconstruction using a gastric tube has been used for esophageal cancer $[2,3]$. However, there is a risk of gastric cancer in the postoperative stomach after surgery if total gastrectomy is not performed [2-4]. Gastric cancer in the postoperative stomach and non-operative stomachs can be detected by follow-up surveillance endoscopy [5]. Endoscopic mucosal resection and endoscopic submucosal dissection (ESD) were performed for early gastric cancer (EGC) with a negligible risk of lymph node metastasis (LNM). The indication for endoscopic resection (ER) was defined according to the current Japanese Gastric Cancer Treatment Guidelines [6]. ESD is the currently accepted treatment for lesions with absolute or expanded indications for ER. Long-term outcomes have been favorable for patients who underwent curative resection $[7,8]$. Similar to ESD of the non-operative stomach (ESD-N) for EGC, ESD of the postoperative stomach (ESD-P) for EGC has been applied to and performed. Several studies reported that ESD-P is an effective and safe treatment modality for EGC and ESD-N [9-12]. Furthermore, it was reported that long-term outcomes of ESD-P for EGC were favorable when curative resection was obtained [11, 12].

However, ESD-P for EGC is known as a technically difficult procedure because of the presence of submucosal fibrosis and staples around the suture line or anastomotic site [9-13]. Some reports have shown high resection rates but with a higher risk of complications with ESD-P than with ESD-N $[14,15]$. However, there were some differences in the baseline characteristics for both groups, which might have affected the outcomes. The rates of tumors in the upper third of the stomach were higher than those of the tumors in the other parts of the stomach after distal gastrectomy, which is also technically difficult [16-19]. Recently, a propensity scorematching analysis has been used to compensate for the differences in the baseline characteristics between the two groups [20-22]. Therefore, the present study aimed to examine the difficulty of ESD-P for EGC compared with ESD-N for EGC by using a propensity score-matching analysis.

\section{Methods \\ Study design}

This was a retrospective, single-center, observational cohort study. We reviewed the medical data from the ESD database, endoscopic reports, and medical records at Kitakyushu Municipal Medical Center (Fukuoka, Japan).

\section{Patients}

ESD was performed to treat 1371 consecutive patients with gastric neoplasms in the non-operative or postoperative stomach between January 1, 2004, and July 31, 2016, at Kitakyushu Municipal Medical Center. One hundred thirty-two patients were excluded from this analysis, because two or more lesions were simultaneously resected by ESD. Furthermore, 193 patients were excluded because the resected specimen was not carcinoma, such as adenoma, according to the current Japanese classification of gastric carcinoma [23]. Therefore, 1046 patients with 1046 lesions were finally included in this study. Forty-one lesions were in the postoperative stomach, whereas 1005 lesions were in the non-operative stomach. Clinicopathological characteristics and clinical outcomes of ESD were collected from the ESD database.

\section{ESD procedures}

All ESD procedures were performed for hospitalized patients. A total of 5-10 mg of midazolam hydrochloride and $15 \mathrm{mg}$ of pentazocine hydrochloride were administered intravenously for sedation just before and during the procedure. A standard single-channel endoscope (GIF-Q260J; Olympus Optical, Tokyo, Japan) was used. VIO 300D or ICC 200 (ERBE Elektromedizin, Tubingen, Germany) was used as the power source for electrical cutting and coagulation. We performed ESD using a standard technique described elsewhere [24-26]. Briefly, marking dots were placed around the lesion using an endo-knife. To lift the lesion, a normal saline solution and hyaluronate sodium with a small amount of epinephrine $(0.001 \mathrm{mg} / \mathrm{mL})$ and $0.8 \%$ indigo carmine were injected in the submucosal layer. Then, a circumferential mucosal incision was created and submucosal dissection was performed with an endo-knife to complete the removal of the lesion. Hemostasis was achieved for bleeding or exposed vessels by using an endo-knife or hemostatic forceps. Insulated tip knife, needle-type knife, or scissor-type knife was 
mainly used as an endo-knife for ESD. Hook knife (KD620; Olympus) was sometimes used during the dissection of the submucosa as a rescue device, which was useful for dissecting the fibrotic site.

\section{Histological evaluation}

After retrieval, ESD specimens were flattened, fixed in $10 \%$ formalin, and sectioned serially at $2-\mathrm{mm}$ intervals. Then, the curability of the specimens was carefully evaluated pathologically according to the Japanese Gastric Cancer Classification [23]. Resections were assessed as curative or non-curative according to the current Japanese gastric cancer treatment guidelines [6]. Non-curative resections were defined as cases that did not meet the curative criteria after ESD.

\section{Clinical outcomes}

The primary outcome of this study was procedure time, which was defined as the time from the beginning of the mucosal incision to resection completion. A prolonged procedure was defined when completion of ESD required more than $60 \mathrm{~min}$ [27]. Secondary outcomes of this study were resection rates (including en bloc, complete, and curative resections) and adverse event rates (including perforation and postprocedural bleeding). A lesion resected in one piece was considered en bloc resection. A lesion resected in an en bloc pattern with tumor-free lateral and vertical margins was considered complete resection. A lesion resected in a complete resection pattern that fulfilled the curative criteria was considered curative resection. Perforations were diagnosed endoscopically during the ESD procedure or by free air during abdominal radiography or CT after ESD. Perforations detected during the ESD procedure were closed with metal clips. Postoperative bleeding was diagnosed as clinical evidence of bleeding after ESD, such as melena and hematemesis with a decrease of $<2 \mathrm{~g} / \mathrm{dL}$ in the hemoglobin level or bleeding confirmed by routine or emergency second-look endoscopy that required transfusion or additional endoscopic hemostasis. We also investigated the proportion of patients who underwent ESD using multiple kinds of endo-knives in both groups.

\section{Statistics}

This was a retrospective study without randomization. Therefore, there were potential confounding biases among the two groups involved. According to previous reports, tumor size, tumor location, depth of tumor invasion, the presence of an ulcer (scar), tumor histology, and skill of the operator were associated with the difficulty of the ESD procedure [16, 18, 19, 27-29]. To balance the bias, we used logistic regression of the following factors for the propensity score calculation [30]: age ( $\geq 75$ years vs $<75$ years), sex (male vs female), tumor size
( $\geq 21 \mathrm{~mm}$ vs $<21 \mathrm{~mm}$ ), tumor location (in the upper third vs in the middle third or lower third of the stomach), depth of tumor invasion (mucosa vs submucosa), tumor histology (differentiated vs undifferentiated), ulcer (scar) (presence vs absence), and operator skill level (expert vs trainee). Previous studies reported that $30-60$ procedures was the cut-off number for the learning curve of ESD $[29,31,32]$. Therefore, experts were defined as those with experience in performing 50 or more ESD procedures, whereas trainees were those with experience in performing fewer than 50 ESD procedures. In this study, there were 28 experts and 13 trainees. Using calipers $(0.2)$ with a width equal to 0.25 of the standard deviation of the logit of the propensity score, we performed propensity score analysis with 1:1 matching using the nearest neighbor matching method. We estimated the area under the receiver-operating characteristics curve, which was 0.77 and thus indicated good predictive power, to validate the model of this study. We evaluated the two groups by using the absolute standardized differences (ASDs) before and after matching to confirm propensity scoring balance. If ASDs were within $1.96 \sqrt{2} / \mathrm{n}$ after matching, then we considered the characteristics to be well-balanced [33]. We analyzed the baseline characteristics and outcomes of this study using the $\chi^{2}$ or Fisher exact tests for categorial data or the Mann-Whitney U test for continuous data not distributed normally. In addition, univariate analysis was conducted to evaluate tumor factors involving long procedure times for ESD-P by using Fisher exact test for the categorical data.

$P<0.05$ was considered statistically significant for all tests. All statistical data analyses were performed using JMP Pro 13.0 software.

\section{Ethics}

We conducted this study in accordance with the Declaration of Helsinki. This study protocol was approved by the institutional review board of Kitakyushu Municipal Medical Center (no. 201701056). Written informed consent was obtained from all patients before treatment in accordance with the institutional protocol. The first and last authors take complete responsibility for the integrity of the data and the accuracy of the data analysis.

\section{Results}

Baseline characteristics before propensity score-matching ESD-P and ESD-N was performed in 41 and 1005 patients, respectively (shown in Fig. 1). The baseline characteristics of 1046 patients are shown in Table 1 . Significantly higher percentages of male patients, those with the original tumor location in the upper third of the stomach, and experts comprised the ESD-P group. There were no significant differences among the two groups regarding other factors. The types of previous 


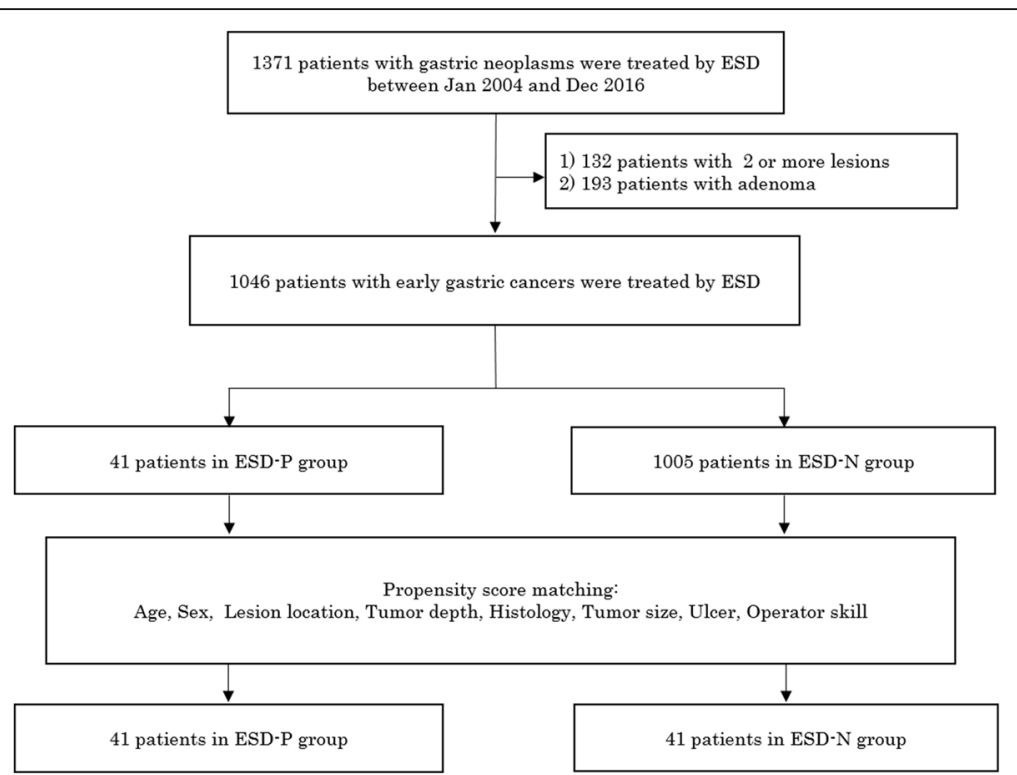

Fig. 1 Flowchart of patients and lesions enrolled in this study. ESD, endoscopic submucosal dissection; ESD-P, endoscopic submucosal dissection of the postoperative stomach; ESD-N, endoscopic submucosal dissection of the non-operative stomach

gastrectomy performed for lesions in the postoperative stomach included 27 distal gastrectomies (65.9\%), 5 proximal gastrectomies $(12.2 \%), 1$ pylorus-preserving gastrectomy $(2.4 \%)$, and 8 esophagectomies (19.5\%).

\section{Matched factors after propensity score-matching}

Propensity score-matching created 41 matched pairs (shown in Fig. 1). All patients in the ESD-P group were matched. The matching factors of the ESD-P and ESD-N groups are shown in Table. 2 . There were no significant differences in any factors among the two groups. In addition, all ASDs were within $1.96 \sqrt{2} / \mathrm{n}$ (0.43) for all matching factors. Therefore, all matched factors after propensity score-matching were well-balanced in this study.

\section{Treatment outcomes after propensity score-matching}

The parameters of treatment outcomes are shown in Table 3. Procedure times for the ESD-P group were significantly longer than those for the ESD-N group $(P<0.001)$. The en bloc resection rate was $100 \%$ for both groups. Complete resection rates were 95.1\% (39/41) for the ESD-P group and 97.6\% (40/41) for the ESD-N group. The curative resection rate was $90.2 \%$ (37/41) for both groups. Regarding adverse events, the perforation rate was $2.4 \%(1 / 41)$ for the ESD-P group and 0\% (0/41) for the ESD-N group. The postprocedural bleeding rate was $2.4 \%$ $(1 / 41)$ for both groups. There were no significant differences regarding treatment outcomes, except for procedure time. However, one patient with perforation during the ESD procedure in the ESD-P group underwent emergency surgery because of postoperative peritonitis. The lesion was located on the suture line. Severe submucosal fibrosis on the suture line was detected during submucosal dissection. Although we used carbon dioxide inflation during the ESD procedure and the site of perforation was closed with metal clips immediately after the ESD procedure, the patient still had peritonitis the next day. Postoperative bleeding occurred in one patient in each group. All cases of postoperative bleeding were successfully managed by endoscopic hemostasis with hemostatic forceps; thus, blood transfusions were not required. According to the pathological evaluation, four patients in each group did not meet the curative criteria after ESD. All four patients in the ESD-P group were followed up without any additional treatment. However, one among these four patients underwent additional surgery because a residual tumor was detected in the specimen resected during the initial surgery.

In addition, the proportion of patients who underwent ESD-P using multiple kinds of endo-knives was higher than that of patients who underwent ESD-N using multiple kinds of endo-knives, but not significant $(36.6 \%$ in ESD-P vs 17.1 in ESD-P, $P=0.080$, shown in Table 3).

Table 4 shows the factors associated with prolonged procedures in the ESD-P group. Tumors involving the suture line or anastomotic site were significantly associated with prolonged procedures $(p=0.038)$, but previous surgery type, size, depth, location, morphology, histology, and presence of an ulcer (scar) in the tumor were not significantly associated with prolonged procedures.

\section{Discussion}

To the best of our knowledge, this is the first study comparing the clinical outcomes between ESD-P and ESD-N 
Table 1 Baseline characteristics of the 1046 patients who underwent ESD before propensity score-matching

\begin{tabular}{|c|c|c|c|c|}
\hline & ESD-P & ESD-N & $p$ & ASD \\
\hline & $n=41$ & $n=1005$ & & \\
\hline Age & & & 0.83 & \\
\hline Mean \pm SD & $70.4 \pm 9.16$ & $71.0 \pm 8.82$ & & \\
\hline Median (range) & $70(65-78)$ & $72(65-77)$ & & 0.834 \\
\hline Sex & & & $0.004^{*}$ & \\
\hline Male & 37 (90.2\%) & 706 (70.2\%) & & 0.14 \\
\hline Female & $4(9.8 \%)$ & $299(29.8 \%)$ & & 0.14 \\
\hline Lesion location & & & $<0.001^{*}$ & \\
\hline$U$ & $13(31.7 \%)$ & $157(15.6 \%)$ & & 0.39 \\
\hline M & $24(58.5 \%)$ & $501(34.5 \%)$ & & 0.50 \\
\hline L & $4(9.8 \%)$ & 347 (49.9\%) & & 0.97 \\
\hline Morphology & & & 0.62 & \\
\hline Protruding & $16(39.1 \%)$ & $322(32.0 \%)$ & & 0.15 \\
\hline Flat & $0(0 \%)$ & $11(1.1 \%)$ & & 0.15 \\
\hline Depressed & $25(61.0 \%)$ & $672(66.9 \%)$ & & 0.12 \\
\hline Histology & & & 0.27 & \\
\hline Differentiated & 39 (95.1\%) & $981(97.6 \%)$ & & 0.13 \\
\hline Undifferentiated & $2(4.9 \%)$ & $24(2.4 \%)$ & & 0.13 \\
\hline Lesion size (mm) & & & 0.06 & \\
\hline Mean \pm SD & $15.0 \pm 7.16$ & $19.8 \pm 13.8$ & & 0.44 \\
\hline Median (range) & $14(4-35)$ & $16(2-115)$ & & \\
\hline Specimen size $(\mathrm{mm})$ & & & 0.56 & \\
\hline Mean \pm SD & $38.7 \pm 8.18$ & $42.4 \pm 15.3$ & & 0.30 \\
\hline Median (range) & $40(16-60)$ & $40(15-140)$ & & \\
\hline Depth & & & 0.50 & \\
\hline pT1a & 37 (90.2\%) & $858(85.4 \%)$ & & 0.15 \\
\hline pT1b & $4(9.8 \%)$ & $147(14.6 \%)$ & & 0.15 \\
\hline Ulcer (scar) & & & 0.76 & \\
\hline Presence & $2(4.9 \%)$ & $80(8.0 \%)$ & & 0.12 \\
\hline Absence & 39 (95.1\%) & 921 (92.0\%) & & 0.12 \\
\hline $\begin{array}{l}\text { Suture line or } \\
\text { anastomotic site }\end{array}$ & & & - & \\
\hline Involving & $19(\%)$ & - & & - \\
\hline Not involving & $22(\%)$ & - & & - \\
\hline Skill level & & & $<0.001^{*}$ & \\
\hline Expert & 36 (87.8\%) & $623(62.0 \%)$ & & 0.62 \\
\hline Trainee & $5(12.2 \%)$ & $382(38.0 \%)$ & & 0.62 \\
\hline
\end{tabular}

$E S D$ endoscopic submucosal dissection, $S D$ standard deviation, $A S D$ absolute standardized differences, $U$ upper third of the stomach, $M$ middle third of the stomach, $L$ lower third of the stomach, pT1a tumor invasion within the mucosa, $p T 1 b$ tumor invasion in the submucosa or deeper

The $p$ value was calculated using the $x^{2}$ test or Fisher exact test for categorical data The $p$ value was calculated using the Mann-Whitney $\mathrm{U}$ test for continuous data not normally distributed

*Significant for the treatment of EGC using a propensity scorematching analysis. Additionally, various types of post-operative stomachs, including not only distal gastrectomy but also proximal gastrectomy, pylorus preserving gastrectomy and esophagectomy, were included and assessed in the present study. Although a high rate of curative resection with a low rate of adverse event for ESD was achieved regardless of the type of post-operative stomach, ESD-P for EGC required significantly longer procedure times than ESD-N for EGC. Moreover, one patient who underwent ESD-P for EGC only on the suture line required emergency surgery.

In this study, $65.9 \%(27 / 41)$ of the patients in the ESD-P group who underwent distal gastrectomy required resection of the middle and lower thirds of the stomach, indicating that EGC of ESD-P frequently originally developed in the upper third of the stomach. It is generally accepted that it is more difficult to complete ESD in the upper third than in the middle third or lower third of the stomach [16-19]. Therefore, it is not appropriate to directly compare the clinical outcomes between ESD-P and ESD-N, because the lesion locations in both procedures significantly differ; thus, we performed a propensity score-matching analysis and included this factor as a covariate-matching factor to compensate for this bias. Therefore, we were able to compare the treatment outcomes for both groups after controlling for potential sources of bias according to the covariates. The tumor location and other covariates did not affect the treatment outcomes of ESD for both groups.

Lesions on the suture line or anastomotic site involve the submucosal fibrosis $[9,13,15]$; therefore, performing ESD for these lesions was difficult to complete. In the present study, 17 lesions had developed on the suture line of the postoperative stomach, whereas 2 lesions on the anastomotic site. Even though ESD of these lesions was performed only by experts, the median ESD time was significantly longer for these lesions than for lesions not involving the suture line or anastomotic site (120.0 min vs $60.5 \mathrm{~min} ; P<0.001$ ). There was no significant difference in the procedure time between lesions on the suture line and those on the anastomotic site $(120.0 \mathrm{~min}$ vs $130 \mathrm{~min}$; $P=0.69$ ). Lesion development on the suture line or anastomotic site was the only independent factor associated with prolonged procedure times (Table 4). In addition, it has been reported that ESD of lesions on the suture line is associated with a high rate of perforation $[9,15]$. In this study, there was only one perforation case during ESD of a lesion located on the suture line in the postoperative stomach, even though experts performed the ESD procedure. Despite the non-negligible risk of perforation and prolonged procedure time, a high rate of curative resection has been achieved, even for lesions that developed on the suture line or anastomotic site of the postoperative 
Table 2 Matching factors between ESD-P and ESD-N after propensity score-matching

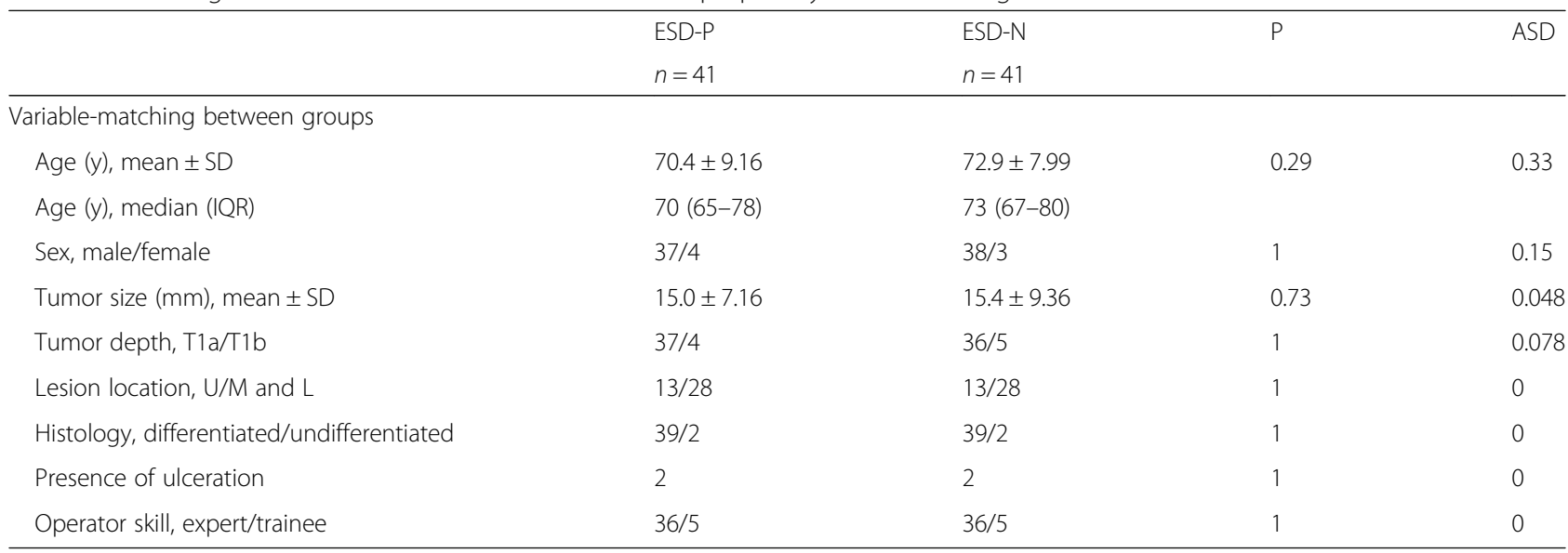

$E S D-P$, endoscopic submucosal dissection of the postoperative stomach, ESD-N endoscopic submucosal dissection of the non-operative stomach, SD standard deviation, $A S D$ absolute standardized differences, $p T 1 a$ tumor invasion within the mucosa, $p T 1 b$ tumor invasion in the submucosa or deeper, $U$ upper third of the stomach, $M$ middle third of the stomach, $L$ lower third of the stomach

The $p$ value was calculated using the $x^{2}$ test or Fisher exact test for the categorical data

The $p$ value was calculated using the Mann-Whitney $\mathrm{U}$ test for continuous data not normally distributed

stomach. The complication rate of ESD-P in the present study was lower than those of previous reports [9-12]. This might be because of the fact that $87.8 \%$ (36/41) of ESD-P procedures were performed by experts. ESD-P should be performed cautiously by highly experienced experts, especially when the lesions are located on the suture line or anastomotic site.

We should take the difference in cost of ESD between the groups into account in addition to the procedure time. The proportion of patients who underwent ESD-P using multiple kinds of endo-knives seems to be higher than that of patients who underwent ESD-N using multiple kinds of endo-knives, although it did not reach to a statistical significance (36.6\% in ESD-P vs $17.1 \%$ in ESD-N; $P=0.080$, shown in Table 3). Therefore, it seemed that ESD-P was costlier than ESD-N in terms of the number of devices used during the procedure.

In this study, consistent with previous reports [9-12], high rates of en bloc and complete resections and resulting high rates of curative resection were achieved not only in the ESD-N group but also in the ESD-P group. All patients with curative resection in both groups had no recurrence. In contrast, there were four patients with non-curative resection in each group. Regarding ESD-N, one of the four patients with non-curative resection underwent additional surgery, which resulted in finding

Table 3 Treatment outcomes for ESD-P and ESD-N after propensity score-matching

\begin{tabular}{llll}
\hline & ESD-P & ESD-N \\
$n=41$ & $n=41$ \\
\hline Procedure time, min, median (IQR) & $85(57-120)$ & $51(28-79)$ & $<0.001^{*}$ \\
$\quad$ Involving suture line, min, median (IQR) & $120.0(89.0-160.0)$ & - & \\
$\quad$ Not involving suture line, min, median (IQR) & $60.5(38.5-84.75)$ & $57.8 \pm 39.4$ \\
Procedure time, min, mean \pm SD & $95.0 \pm 59.0$ & $16(39.0 \%)$ & $41(100 \%)$ \\
Long procedure time & $30(73.2 \%)$ & $40(97.6 \%)$ & - \\
En bloc resection, $n(\%)$ & $41(100 \%)$ & $37(90.2 \%)$ & $0.004^{*}$ \\
Complete resection, $\mathrm{n}(\%)$ & $39(95.1 \%)$ & $0(0 \%)$ & 1 \\
Curative resection, $\mathrm{n}(\%)$ & $37(90.2 \%)$ & $1(2.4 \%)$ & 1 \\
Perforation, $\mathrm{n}$ (\%) & $1(2.4 \%)$ & $7(17.1 \%)$ \\
Postprocedure bleeding, $\mathrm{n}(\%)$ & $1(2.4 \%)$ & $15(36.6 \%)$ & 1 \\
Use of multiple kinds of endo-knives, $\mathrm{n}(\%)$ & 0.080 \\
\hline
\end{tabular}

ESD-P, endoscopic submucosal dissection of the postoperative stomach; ESD-N, endoscopic submucosal dissection of the non-operative stomach; SD, standard deviation; IQR, interquartile range

The $p$ value was calculated using the $x^{2}$ test or Fisher exact test for categorical data

The $p$ value was calculated using the Mann-Whitney $U$ test for continuous data not normally distributed

*Significant value 
Table 4 Tumor factors involving long procedure times for ESD-P

\begin{tabular}{|c|c|c|c|}
\hline & $\begin{array}{l}\text { Long procedure } \\
\text { time }\end{array}$ & $\begin{array}{l}\text { Shorter procedure } \\
\text { time }\end{array}$ & $P$ \\
\hline & $n=30$ & $n=11$ & \\
\hline Previous surgery type, (\%) & & & 0.412 \\
\hline Distal gastrectomy & $20(66.7 \%)$ & $7(63.6 \%)$ & \\
\hline Proximal gastrectomy & $3(10.0 \%)$ & $2(18.2 \%)$ & \\
\hline $\begin{array}{l}\text { Pylorus preserving } \\
\text { gastrectomy }\end{array}$ & $0(0 \%)$ & $1(9.1 \%)$ & \\
\hline Esophagectomy & $7(23.3 \%)$ & $1(9.1 \%)$ & \\
\hline Tumor size, n (\%) & & & 0.651 \\
\hline$<21$ & $24(80 \%)$ & $10(90.9 \%)$ & \\
\hline$\geq 21$ & $6(20 \%)$ & $1(9.1 \%)$ & \\
\hline Tumor depth, n (\%) & & & 0.559 \\
\hline T1a & $26(86.7 \%)$ & $11(100 \%)$ & \\
\hline $\mathrm{T} 1 \mathrm{~b}$ & $4(13.3 \%)$ & $0(0 \%)$ & \\
\hline Lesion location, n (\%) & & & 0.719 \\
\hline Upper & $9(30.0 \%)$ & $4(36.4 \%)$ & \\
\hline Middle or lower & $21(70.0 \%)$ & $7(63.6 \%)$ & \\
\hline $\begin{array}{l}\text { Suture line or anastomotic } \\
\text { site, } n(\%)\end{array}$ & & & $0.038^{*}$ \\
\hline Involved & $17(56.7 \%)$ & $2(18.2 \%)$ & \\
\hline Not involved & $13(43.4 \%)$ & $9(81.8 \%)$ & \\
\hline Morphology, n (\%) & & & 0.287 \\
\hline Flat or depressed & $20(66.7 \%)$ & $5(45.5 \%)$ & \\
\hline Protruding & $10(33.3 \%)$ & $6(54.5 \%)$ & \\
\hline Histology, n (\%) & & & 1 \\
\hline Differentiated & $28(93.3 \%)$ & $11(100 \%)$ & \\
\hline Undifferentiated & $2(6.7 \%)$ & $0(0 \%)$ & \\
\hline Ulceration (scar) & & & 1 \\
\hline Presence & $2(6.7 \%)$ & $0(0 \%)$ & \\
\hline Absence & $28(93.3 \%)$ & $11(100 \%)$ & \\
\hline
\end{tabular}

ESD-P endoscopic submucosal dissection for early gastric cancer in the postoperative stomach

The $p$ value was calculated using the $x^{2}$ test or the Fisher exact test for categorical data

*Significant

a remnant tumor of the resected specimen. The remaining patients did not undergo any additional treatments, and all had no recurrence. According to the current guidelines [6], additional surgery is indicated for all patients with non-curative ESD resection and significant risk for LNM. However, it has been reported that almost half of the EGC patients with non-curative ESD resection did not undergo additional surgery due to their advanced age or comorbidities [34]. Furthermore, it has been reported that LNM was found in only $5-10 \%$ of patients with such lesions in the non-operative stomach [35]. Regarding ESD-P, all four patients who underwent non-curative ESD resection were followed up without any additional treatments. The risk of
LNM in T1 remnant cancer after distal gastrectomy resection was reported to be only $2.4 \%(1 / 42)$ [36]. Patients who underwent non-curative ESD resection in the remnant stomach refused radical completion gastrectomy of the remnant stomach due to the high risk of complications and postoperative mortality rates (from 13 to 41\%) [37-40]. Completion gastrectomy for EGC of the remnant stomach is much riskier during the perioperative period compared to that of the non-remnant stomach [3740]. Given the few data regarding the prognosis of patients with non-curative ESD resection for the postoperative stomach, it should be considered whether additional surgery should be performed based on the current guidelines due to the non-negligible risk of postoperative mortality for radical completion gastrectomy of the remnant stomach. As a result, all four patients with non-curative ESD resection in the ESD-P group had no recurrence. A recent report suggested a useful scoring system for evaluating the risk of LNM for non-curative patients [41]. Using this scoring system, 3 patients who underwent non-curative ESD-P were classified as low-risk patients, with an estimated LNM risk of 2-3\%. The other patient was classified as having an intermediate risk, with estimated LNM risk of $6-7 \%$. In this study, there were no high-risk patients. This system may be useful for deciding additional surgery even for patients who underwent non-curative ESD-P. However, some lymph nodes might be resected in patients who underwent the previous surgery for a malignant lesion. This system may not be applicable to non-curative patients with post-operative stomach. In the future, further expanded criteria or more appropriate risk stratifications may be required for ESD-P for EGC.

This study had some limitations. First, this was a singlecenter retrospective study with a relatively small sample size. Second, we did not consider the use of traction method, such as a traction method using dental floss and a hemoclip, which was recently reported to shorten the procedure time of ESD [42]. Third, we did not consider medications, especially anti-thrombotic drugs, in this study, since they were discontinued during the perioperative period, because the patients enrolled in this study had undergone ESD treatments before the current Japanese guidelines for the use of anti-thrombotic agents were established [43]. Hence, there are no data regarding the influence of anti-thrombotic agents on ESD-P. Fourth, we could not deny the possibility that the learning curve might affect the technical outcome of this present study, which could not be fully compensated by the propensity score-matching. Fifth, ESD-P was performed by only 9 experts, specifically 8 experts who performed ESD-P involving the suture line or anastomotic site, whereas, 8 experts performed ESD-N after propensity score-matching. Seven experts were in common among ESD-P and ESD-N after matching Furthermore, 6 experts were in common among 
ESD-P involving suture line or anastomic site and ESD-N after matching. Therefore, there seems to be some bias of operators. Sixth, we did not consider the difference of the electrosurgical units (VIO300D or ICC200) used in the ESD procedures, which might have affected the study outcome. A larger prospective, randomized, controlled trial will be required to determine further treatment guidelines, including the influence of anti-thrombotic agents, for ESD-P.

\section{Conclusion}

In conclusion, ESD-P was a more time-consuming procedure than ESD-N. However, ESD-P and ESD-N for the treatment of EGC achieved high rates of curative resection with low rates of adverse events. Therefore, ESD could be selected as the treatment for EGC, even in the postoperative stomach, but careful attention must be focused on lesions on the suture line or anastomotic site.

\section{Abbreviations}

ASD: Absolute standardized differences; CT: Computed tomography; EGC: Early gastric cancer; ER: Endoscopic resection; ESD: Endoscopic submucosal dissection; ESD-N: Endoscopic submucosal dissection of the non-operative stomach; ESD-P: Endoscopic submucosal dissection of the postoperative stomach; LNM: Lymph node metastasis

\section{Funding}

No specific grants from any funding agencies were received for this study.

\section{Availability of data and materials}

The datasets used and analyzed during the current study are available from the corresponding author on reasonable request.

\section{Authors' contributions}

ME, SS, and SA: conception and design, drafting of the article, and critical revision of the article for important intellectual content. YH, AY, TH, ST, YM, $\mathrm{YH}$, and $\mathrm{HO}$ : analysis and interpretation of the data. HA: critical revision of the manuscript. El and YO: study supervision. All authors have read and approved the final manuscript.

\section{Ethics approval and consent to participate}

We conducted this study in accordance with the Declaration of Helsinki. This study protocol was approved by the institutional review board of the Kitakyushu Municipal Medical Center on November 18, 2016 (no. 201701056). Written informed consent was obtained from all patients before treatment in accordance with the institutional protocol.

\section{Consent for publication}

Not applicable.

\section{Competing interests}

The authors declare that they have no competing interests.

\section{Publisher's Note}

Springer Nature remains neutral with regard to jurisdictional claims in published maps and institutional affiliations.

\footnotetext{
Author details

'Division of Gastroenterology and Hepatology, Department of Medicine, Nihon University School of Medicine, 1-6 Kanda-Surugadai, Chiyoda-ku, Tokyo 101-8309, Japan. ²Department of Gastroenterology, Kitakyushu Municipal Medical Center, 2-1-1 Bashaku, Kokurakita-ku, Kitakyushu, Fukuoka 802-0077, Japan. ${ }^{3}$ Department of Medicine and Bioregulatory Science, Graduate School of Medical Sciences, Kyushu University, 3-1-1 Maidashi, Higashi-ku, Fukuoka 812-8582, Japan. ${ }^{4}$ Department of Anatomic Pathology,
}

Graduate School of Medical Sciences Kyushu University, 3-1-1 Maidashi, Higashi-ku, Fukuoka 812-8582, Japan.

Received: 28 May 2018 Accepted: 31 July 2018

Published online: 06 August 2018

\section{References}

1. Wang YR, Richter JE, Dempsey DT. Trends and outcomes of hospitalizations for peptic ulcer disease in the United States, 1993 to 2006. Ann Surg. 2010;251:51-8.

2. Allum WH, Stenning SP, Bancewicz J, Clark PI, Langley RE. Long-term results of a randomized trial of surgery with or without preoperative chemotherapy in esophageal cancer. J Clin Oncol. 2009;27:5062-7.

3. Ando N, Kato H, Igaki H, Shinoda M, Ozawa S, Shimizu H, et al. A randomized trial comparing postoperative adjuvant chemotherapy with cisplatin and 5-fluorouracil versus preoperative chemotherapy for localized advanced squamous cell carcinoma of the thoracic esophagus (JCOG9907). Ann Surg Oncol. 2012:19:68-74.

4. Kaneko K, Kondo H, Saito D, Shirao K, Yamaguchi H, Yokota T, et al. Early gastric stump cancer following distal gastrectomy. Gut. 1998:43:342-4.

5. Hosokawa O, Kaizaki Y, Watanabe K, Hattori M, Douden K, Hayashi H, et al. Endoscopic surveillance for gastric remnant cancer after early cancer surgery. Endoscopy. 2002;34:469-73

6. Japanese Gastric Cancer A. Japanese gastric cancer treatment guidelines 2014 (ver. 4). Gastric Cancer. 2017;20:1-19

7. Tanabe S, Ishido K, Matsumoto T, Kosaka T, Oda I, Suzuki H, et al. Long-term outcomes of endoscopic submucosal dissection for early gastric cancer: a multicenter collaborative study. Gastric Cancer. 2017:20:45-52.

8. Suzuki H, Oda I, Abe S, Sekiguchi M, Mori G, Nonaka S, et al. High rate of 5-year survival among patients with early gastric cancer undergoing curative endoscopic submucosal dissection. Gastric Cancer. 2016;19:198-205.

9. Ojima T, Takifuji K, Nakamura M, Nakamori M, Katsuda M, lida T, et al. Endoscopic submucosal dissection for gastric tumors in various types of remnant stomach. Endoscopy. 2014;46:645-9.

10. Takenaka R, Kawahara Y, Okada H, Tsuzuki T, Yagi S, Kato J, et al. Endoscopic submucosal dissection for cancers of the remnant stomach after distal gastrectomy. Gastrointest Endosc. 2008:67:359-63.

11. Nonaka S, Oda I, Makazu M, Haruyama S, Abe S, Suzuki H, et al. Endoscopic submucosal dissection for early gastric cancer in the remnant stomach after gastrectomy. Gastrointest Endosc. 2013;78:63-72.

12. Nonaka S, Oda I, Sato C, Abe S, Suzuki H, Yoshinaga S, et al. Endoscopic submucosal dissection for gastric tube cancer after esophagectomy. Gastrointest Endosc. 2014;79:260-70.

13. Tanaka S, Toyonaga T, Morita Y, Fujita T, Yoshizaki T, Kawara F, et al. Endoscopic submucosal dissection for early gastric cancer in anastomosis site after distal gastrectomy. Gastric Cancer. 2014;17:371-6.

14. Lee JY, Choi IJ, Cho SJ, Kim CG, Kook MC, Lee JH, et al. Endoscopic submucosal dissection for metachronous tumor in the remnant stomach after distal gastrectomy. Surg Endosc. 2010;24:1360-6.

15. Nishide N, Ono H, Kakushima N, Takizawa K, Tanaka M, Matsubayashi H, et al. Clinical outcomes of endoscopic submucosal dissection for early gastric cancer in remnant stomach or gastric tube. Endoscopy. 2012:44:577-83.

16. Imagawa A, Okada H, Kawahara Y, Takenaka R, Kato J, Kawamoto H, et al. Endoscopic submucosal dissection for early gastric cancer: results and degrees of technical difficulty as well as success. Endoscopy. 2006;38: 987-90.

17. Isomoto H, Shikuwa S, Yamaguchi N, Fukuda E, Ikeda K, Nishiyama H, et al. Endoscopic submucosal dissection for early gastric cancer: a large-scale feasibility study. Gut. 2009;58:331-6.

18. Yoon JY, Shim CN, Chung SH, Park W, Chung H, Lee H, et al. Impact of tumor location on clinical outcomes of gastric endoscopic submucosal dissection. World J Gastroenterol. 2014;20:8631-7.

19. Ahn JY, Choi KD, Choi JY, Kim MY, Lee JH, Choi KS, et al. Procedure time of endoscopic submucosal dissection according to the size and location of early gastric cancers: analysis of 916 dissections performed by 4 experts. Gastrointest Endosc. 2011;73:911-6

20. Suzuki S, Gotoda T, Hatta W, Oyama T, Kawata N, Takahashi A, et al Survival benefit of additional surgery after non-curative endoscopic submucosal dissection for early gastric cancer: a propensity score matching analysis. Ann Surg Oncol. 2017;24:3353-60. 
21. Suzuki S, Gotoda T, Kusano C, Iwatsuka K, Moriyama M. The efficacy and tolerability of a triple therapy containing a potassium-competitive acid blocker compared with a 7-day PPI-based low-dose clarithromycin triple therapy. Am J Gastroenterol. 2016;111:949-56.

22. Esaki M, Suzuki S, Hayashi Y, Yokoyama A, Abe S, Hosokawa T, et al. Splash M-knife versus flush knife BT in the technical outcomes of endoscopic submucosal dissection for early gastric cancer: a propensity score matching analysis. BMC Gastroenterol. 2018;18:35.

23. Japanese Gastric Cancer A. Japanese classification of gastric carcinoma: 3rd English edition. Gastric Cancer. 2011;14:101-12.

24. Soetikno R, Kaltenbach T, Yeh R, Gotoda T. Endoscopic mucosal resection for early cancers of the upper gastrointestinal tract. J Clin Oncol. 2005;23:4490-8.

25. Rembacken BJ, Gotoda T, Fujii T, Axon AT. Endoscopic mucosal resection. Endoscopy. 2001;33:709-18.

26. Gotoda T. Endoscopic resection of early gastric cancer. Gastric Cancer. 2007:10:1-11.

27. Kim JH, Nam HS, Choi CW, Kang DH, Kim HW, Park SB, et al. Risk factors associated with difficult gastric endoscopic submucosal dissection: predicting difficult ESD. Surg Endosc. 2017;31:1617-26.

28. Chung IK, Lee JH, Lee SH, Kim SJ, Cho JY, Cho WY, et al. Therapeutic outcomes in 1000 cases of endoscopic submucosal dissection for early gastric neoplasms: Korean ESD study group multicenter study. Gastrointest Endosc. 2009;69:1228-35.

29. Choi IJ, Kim CG, Chang HJ, Kim SG, Kook MC, Bae JM. The learning curve for EMR with circumferential mucosal incision in treating intramucosal gastric neoplasm. Gastrointest Endosc. 2005:62:860-5.

30. D'Agostino RB Jr. Propensity score methods for bias reduction in the comparison of a treatment to a non-randomized control group. Stat Med. 1998;17:2265-81.

31. Yoshida M, Kakushima N, Mori K, Igarashi K, Kawata N, Tanaka M, et al. Learning curve and clinical outcome of gastric endoscopic submucosal dissection performed by trainee operators. Surg Endosc. 2017;31:3614-22.

32. Hong KH, Shin SJ, Kim JH. Learning curve for endoscopic submucosal dissection of gastric neoplasms. Eur J Gastroenterol Hepatol. 2014;26:949-54.

33. Austin PC. Balance diagnostics for comparing the distribution of baseline covariates between treatment groups in propensity-score matched samples. Stat Med. 2009;28:3083-107.

34. Hatta W, Gotoda T, Oyama T, Kawata N, Takahashi A, Yoshifuku Y, et al. Is radical surgery necessary in all patients who do not meet the curative criteria for endoscopic submucosal dissection in early gastric cancer? A multi-center retrospective study in Japan. J Gastroenterol. 2017;52:175-84.

35. Son SY, Park JY, Ryu KW, Eom BW, Yoon HM, Cho SJ, et al. The risk factors for lymph node metastasis in early gastric cancer patients who underwent endoscopic resection: is the minimal lymph node dissection applicable? A retrospective study. Surg Endosc. 2013;27:3247-53.

36. Ohashi M, Katai H, Fukagawa T, Gotoda T, Sano T, Sasako M. Cancer of the gastric stump following distal gastrectomy for cancer. Br J Surg. 2007;94:92-5.

37. Sasako M, Maruyama K, Kinoshita T, Okabayashi K. Surgical treatment of carcinoma of the gastric stump. Br J Surg. 1991;78:822-4.

38. Piso P, Meyer HJ, Edris C, Jahne J. Surgical therapy of gastric stump carcinoma--a retrospective analysis of 109 patients. Hepato-Gastroenterology. 1999:46:2643-7.

39. Lissens P, Filez L, Aerts R, D'Hoore A, Van Cutsem E, Ectors N, et al. Surgery for gastric remnant carcinoma following Billroth II gastrectomy. Eur J Surg Oncol. 1997;23:518-21.

40. Sugiura T, Kato H, Tachimori Y, Igaki H, Yamaguchi H, Nakanishi Y. Second primary carcinoma in the gastric tube constructed as an esophageal substitute after esophagectomy. J Am Coll Surg. 2002;194:578-83.

41. Hatta W, Gotoda T, Oyama T, Kawata N, Takahashi A, Yoshifuku Y, et al. A scoring system to stratify curability after endoscopic submucosal dissection for early gastric cancer: "eCura system". Am J Gastroenterol. 2017;112:874-81.

42. Suzuki S, Gotoda T, Kobayashi Y, Kono S, Iwatsuka K, Yagi-Kuwata N, et al. Usefulness of a traction method using dental floss and a hemoclip for gastric endoscopic submucosal dissection: a propensity score matching analysis (with videos). Gastrointest Endosc. 2016;83:337-46.

43. Fujimoto K, Fujishiro M, Kato M, Higuchi K, Iwakiri R, Sakamoto C, et al. Guidelines for gastroenterological endoscopy in patients undergoing antithrombotic treatment. Dig Endosc. 2014;26:1-14.

\section{Ready to submit your research? Choose BMC and benefit from:}

- fast, convenient online submission

- thorough peer review by experienced researchers in your field

- rapid publication on acceptance

- support for research data, including large and complex data types

- gold Open Access which fosters wider collaboration and increased citations

- maximum visibility for your research: over $100 \mathrm{M}$ website views per year

At BMC, research is always in progress.

Learn more biomedcentral.com/submissions 